\title{
Application of Fourier Transform Near-Infrared (FT-NIR) and Fourier Transform Infrared (FT-IR) Spectroscopy Coupled with Wavelength Selection for Fast Discrimination of Similar Color of Tuber Flours
}

\author{
Rudiati Evi Masithoh ${ }^{1, *}$, Hanim Zuhrotul Amanah ${ }^{1,2}$, and Byoung Kwan Cho ${ }^{2}$ \\ ${ }^{1}$ Department of Agricultural and Biosystems Engineering, Faculty of Agricultural Technology, Universitas Gadjah Mada, \\ Yogyakarta 55281, Indonesia \\ ${ }^{2}$ Department of Biosystems Machinery Engineering, College of Agricultural and Life Science, Chungnam National University, \\ 99 Daehak-ro, Yuseoung-gu, Daejeon 305-764, Republic of Korea
}

\section{* Corresponding author:}

tel: $+62-85743596391$

email:evi@ugm.ac.id

Received: July 25, 2019

Accepted: November 10, 2019

DOI: $10.22146 /$ ijc. 48092

\begin{abstract}
This research aimed at providing a fast and accurate method in discriminating tuber flours having similar color by using Fourier transform near-infrared (FT-NIR) and Fourier transform infrared (FT-IR) spectroscopy in order to minimize misclassification if using human eye, or to avoid adulteration. Reflectance spectra of three types of tubers (consisted of Canna edulis, modified cassava, and white sweet potato) were collected to develop a multivariate model of partial least-squares discriminant analysis (PLS-DA). Several spectra preprocessing methods were applied to obtain the best calibration and prediction model, while variable importance in the projection (VIP) wavelength selection method was used to reduce variables in developing the model. The PLS-DA model achieved 100\% accuracy in predicting all types of flours, both for FT-NIR and FT-IR. The model was also able to discriminate all flours with coefficient of determination $\left(R^{2}\right)$ of 0.99 and a standard error of prediction (SEP) of $0.03 \%$ by using $1^{\text {st }}$ Savitzky Golay (SG) derivative method for the FT-NIR data, as well as $R^{2}$ of 0.99 and SEP of $0.08 \%$ by using $1^{\text {st }}$ Savitzky Golay (SG) derivative method for the FT-IR data. By applying the VIP method, the variables were reduced from 1738 to 608 variables with $R^{2}$ of 0.99 and SEP of $0.09 \%$ for FT IR and from 1557 to 385 variables with $R^{2}$ of 0.99 and SEP of $0.05 \%$ for FT NIR.
\end{abstract}

Keywords: FT-NIR; FT-IR; VIP; PLS-DA; tuber flour

\section{- INTRODUCTION}

Tubers, such as cassava, sweet potato, arrowroot, taro, and Canna edulis, are abundantly cultivated in several regions in Indonesia and usually consumed as a staple food to alternate rice. Those crops are rich in carbohydrate, providing about $1 / 3$ of that of an equivalent weight of rice or wheat due to their high moisture content, and also sources of a number of compounds such as saponins, phenolic compounds, glycoalkaloids, phytic acids, carotenoids, and ascorbic acid [1]. Tubers have a high metabolic activity after harvest; therefore, they are more perishable than grains [2]. One method to prolong the tuber self-life is by making them into flours. In flour form, the crops can be utilized for several food productions such as for noodles, biscuits, snacks, or bread.

Among the tubers, Canna edulis, modified cassava, and white sweet potato have white color in flour form. Their colors are similar, making them difficult to be differentiated by the human eye. Although similar in color, those flours are different in composition and also in price. Chemical compositions are required to determine the method in food processing as well as to provide nutrition information for people susceptible to certain compounds. Due to the difference in price, adulteration of flours is sometimes inevitable. Low quality and price products are added to other products for economic reasons [3]. Based on seller price in 
Indonesia, white sweet potato has the most expensive price, followed by Canna edulis and modified cassava flours. Therefore, an incorrect judgment of the type of flours may bring about health or economic consequences.

Quality determination of flours is usually done by sensory analysis using trained panelists [4], GC-MS for aroma [5], colorimeter, and spectrophotometer for color [6], as well as quantitative analysis in the laboratory [7]. Those methods require intense sample preparation, timeconsuming, and skilled labors, especially for sensory analysis. For massive and large products, fast and rapid discrimination methods are needed.

Spectroscopy is now becoming popular as a fast and rapid method for qualitative and quantitative food analysis. Infrared spectroscopic covers wide range of electromagnetic regions consisting of near-infrared (NIR) at $14000-4000 \mathrm{~cm}^{-1}$, mid-infrared (MIR) at $4000-400 \mathrm{~cm}^{-1}$ and far infrared at $400-50 \mathrm{~cm}^{-1}$; however, MIR and NIR regions are commonly used for food quality analysis [8]. Whereas spectra in the MIR region contain information on the fundamental molecular vibrations, the absorption spectra in the NIR region contain information on the overtones and combinations of fundamental vibrations [9]. MIR spectroscopy is able to identify very similar and complex molecular structures, so it is useful for compound identification, while NIR spectroscopy is able to identify molecular interactions and chemical groups [10].

Both NIR and IR spectroscopy have been applied for quality identification of flour from wheat [11], rice [12], cassava, taro, and yam [13], and sweet potato [14]. Other applications combine chemometrics analysis with NIR and IR spectroscopy for detecting adulteration of flour products [15-16]. Classification of products using NIR and IR spectroscopy are also studied [17-18], but study about distinguishing flours that have a similar color has not been conducted. Therefore, this paper aims at discriminating flours from tuber crops that have similar color by applying Fourier transform near-infrared (FTNIR) and Fourier transform infrared (FT-IR) spectroscopy. This study also applies variable importance in the projection (VIP) wavelength selection method in order to reduce processing time.

\section{- EXPERIMENTAL SECTION}

\section{Materials}

Canna edulis, modified cassava, and white sweet potato flours were purchased from ten different sellers to obtain large varieties of samples. The sample of this study was Canna edulis, modified cassava, and white sweet potato flours obtained from several farmers in Indonesia to provide a wide range of variety shown from different brands. There were ten brands for each flour, making in a total of 30 batches of flour. For every batch, 3 samples were taken for spectra analysis, making a total of 90 spectra data.

\section{Instrumentation}

FT-NIR spectrophotometer (Antaris II FT-NIR analyzer, Thermo Scientific Co., Waltham, MA, USA) assembled with an InGaAs detector module, beam splitter, sample holder, moving mirror, laser diode, and halogen NIR light source, as well as FT-IR spectrometer (Nicolet 6700, Thermo Scientific Co., Waltham, MA, USA) using an attenuated total reflectance (ATR) sampling technique, a deuterated triglycine sulfate (DTGS) detector, and a KBr beam splitter, were used for spectra measurement.

\section{Procedure}

\section{FT-NIR spectra acquisition}

The reflectance spectra of 90 flours were acquired using the FT-NIR spectrophotometer (Antaris II FTNIR analyzer, Thermo Scientific Co., Waltham, MA, USA) assembled with an InGaAs detector module, beam splitter, sample holder, moving mirror, laser diode, and halogen NIR light source. Each sample was scanned for 32 scans at the wavelength range of $4000-10,000 \mathrm{~cm}^{-1}$ at $4 \mathrm{~cm}^{-1}$ spectral resolution. The background scan was conducted with a golden slit frequently before acquiring the spectrum from each flour. In total, there were 1557 variables for FT-NIR.

\section{FT-IR spectra acquisition}

The spectra of 90 flours were obtained using the Nicolet 6700 FT-IR spectrometer with a range of 650$4000 \mathrm{~cm}^{-1}$, using an attenuated total reflectance (ATR) 
sampling technique, a highly sensitive deuterated triglycine sulfate (DTGS) detector, and a $\mathrm{KBr}$ beam splitter. A total of 32 successive scans of each sample with a spectral resolution of $3 \mathrm{~cm}^{-1}$ was recorded from the bare diamond ATR crystal. A background was recorded before every group of samples scanned with a clean ATR crystal. In total, there were 1738 variables for FT-IR.

\section{Spectra pre-treatment and analysis}

All spectra collections were done using Thermo Scientific $^{\text {tix }}$ OMNIC $^{\text {mi }}$ Series Software. The spectra data were transformed to MS Excel 2013 and then imported to the Matlab version R2018a (The MathWorks, Inc., Natick, MA, USA) for spectra preprocessing and chemometrics analysis. Two multivariate data analysis techniques were applied, namely principal component analysis (PCA) and partial least-squares discriminant analysis (PLS-DA), to develop a classification model for Canna edulis, modified cassava, and white sweet potato flours. PCA was used for reducing dimensions and visualizing data distribution concerning discrimination of samples. For developing the PLS-DA model, the FT-NIR and FT-IR spectra were preprocessed through normalization methods (minimum, maximum, and range), standard normal variate transformation (SNV), multiple scatter correlation (MSC), and Savitzky-Golay (SG) smoothing ( $1^{\text {st }}$ and $2^{\text {nd }}$ derivatives). The $3^{\text {rd }}$ order of polynomial fit and 5 smoothing points were used for SG smoothing. Variable importance in the projection (VIP) wavelength selection method was used to reduce variables in developing the model. Of 90 spectra data, $60 \%$ and $40 \%$ of the data were used for calibration and prediction, respectively. A full-cross validation method (leave-oneout) was used in developing a calibration model using the PLS-DA method. The coefficient of determination $\left(\mathrm{R}^{2}\right)$ and a standard error of prediction (SEP) were used to justify the accuracy of the model.

\section{- RESULTS AND DISCUSSION}

The analysis results from the spectra obtained using FT-NIR and FT-IR were used to compare the performance accuracy of the two spectroscopic techniques in distinguishing Canna edulis, modified cassava, and white sweet potato flours.

\section{Spectra Data Exploration}

Original spectra of all flours collected from FTNIR and FT-IR systems are presented in Fig. 1. Fig. 1(a) shows the FT-NIR in the wavenumber range of 4000$10000 \mathrm{~cm}^{-1}$ in which all spectra are widely distributed between samples and have a similar pattern. However, the relative absorbance of Canna edulis flour is characterized by higher relative absorbance compared to other flours. Although not too distinct, several peaks on FT-NIR spectra can be observed, which may reflect particular chemical compounds. Original spectra of flours acquired from FT-IR at a wavenumber of 650$4000 \mathrm{~cm}^{-1}$ (Fig. 1(b)) shows sharper fundamental absorptions; therefore, they can be easily assigned to specific chemical compounds [19].

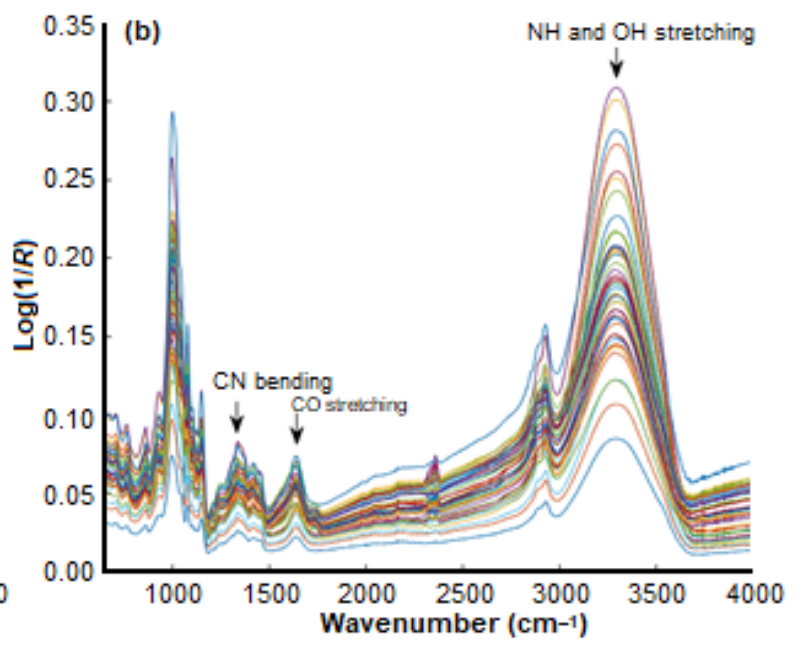

Fig 1. Original spectra of (a) FT-NIR and (b) FT-IR 
The typical absorptions in the NIR region (Fig. 1(a)) are dominated by $\mathrm{CH}$ second overtone bands (7692$\left.8695 \mathrm{~cm}^{-1}\right), \mathrm{NH}$ and $\mathrm{OH}$ first overtone bands (5882$\left.7142 \mathrm{~cm}^{-1}\right), \mathrm{CH}$ first overtone bands $\left(5882-7142 \mathrm{~cm}^{-1}\right)$, $\mathrm{NH}$, and $\mathrm{OH}$ combination bands $\left(4444-5263 \mathrm{~cm}^{-1}\right)$ and $\mathrm{CH}$ combination bands $\left(4166-4444 \mathrm{~cm}^{-1}\right)$ [17]. Fig. 1(b) shows the FT-IR spectra of Canna edulis, modified cassava, and white sweet potato. In general, FT-IR spectra can be classified into two regions: below $1500 \mathrm{~cm}^{-1}$, which are the fingerprint region, and $1500-4000 \mathrm{~cm}^{-1}$, known as the functional group region [20]. Absorption peaks at a range of $3000-3500 \mathrm{~cm}^{-1}$ show $\mathrm{NH}$ and $\mathrm{OH}$ stretching, while at 1456 and $1643 \mathrm{~cm}^{-1}$ show $\mathrm{CN}$ bending and $\mathrm{CO}$ stretching, respectively [21].

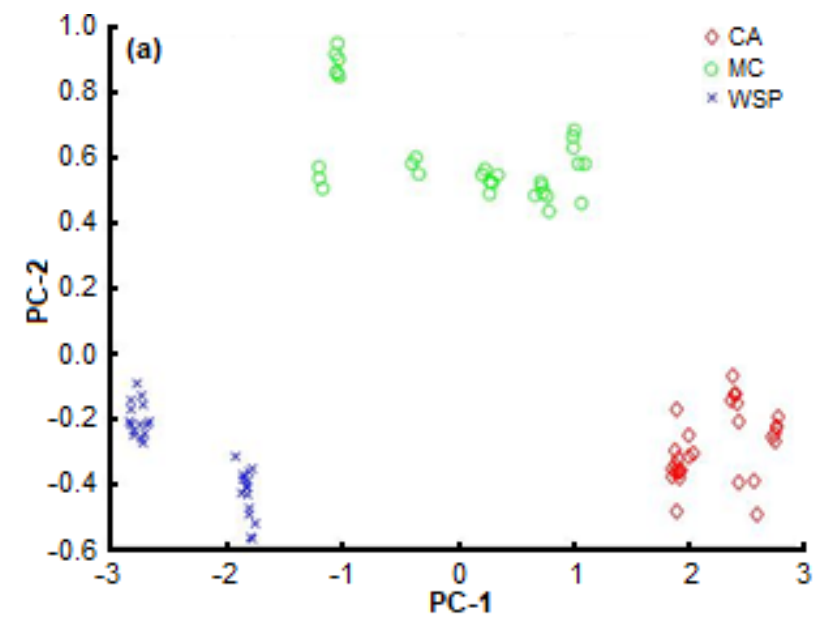

\section{Principle Component Analysis}

The Principal Component Analysis (PCA) is applied for dimensional reduction and data exploration of the three tuber flours. By using original spectra for both FTNIR and FT-IR data, the flours are clearly classified, as shown in Fig. 2(a) and Fig. 3(b). The loading plots (Fig. 2(b) and Fig. 3(b)) provide information about absorption bands, which largely contribute to the discrimination of flours according to the $\mathrm{PC}$ values.

As illustrated in Fig. 2(a), the plot of PC-1 and PC2 provides better visualization of discrimination among the groups of samples for FT-NIR data. PC-1 and PC-2 altogether account for $99.5 \%$ the total variability. The PC

Fig 2. PCA of FT-NIR spectra of CA (Canna edulis), MC (modified cassava), and WSP (white sweet potato): (a) score plot and (b) loadings plot of PC-1 and PC-2
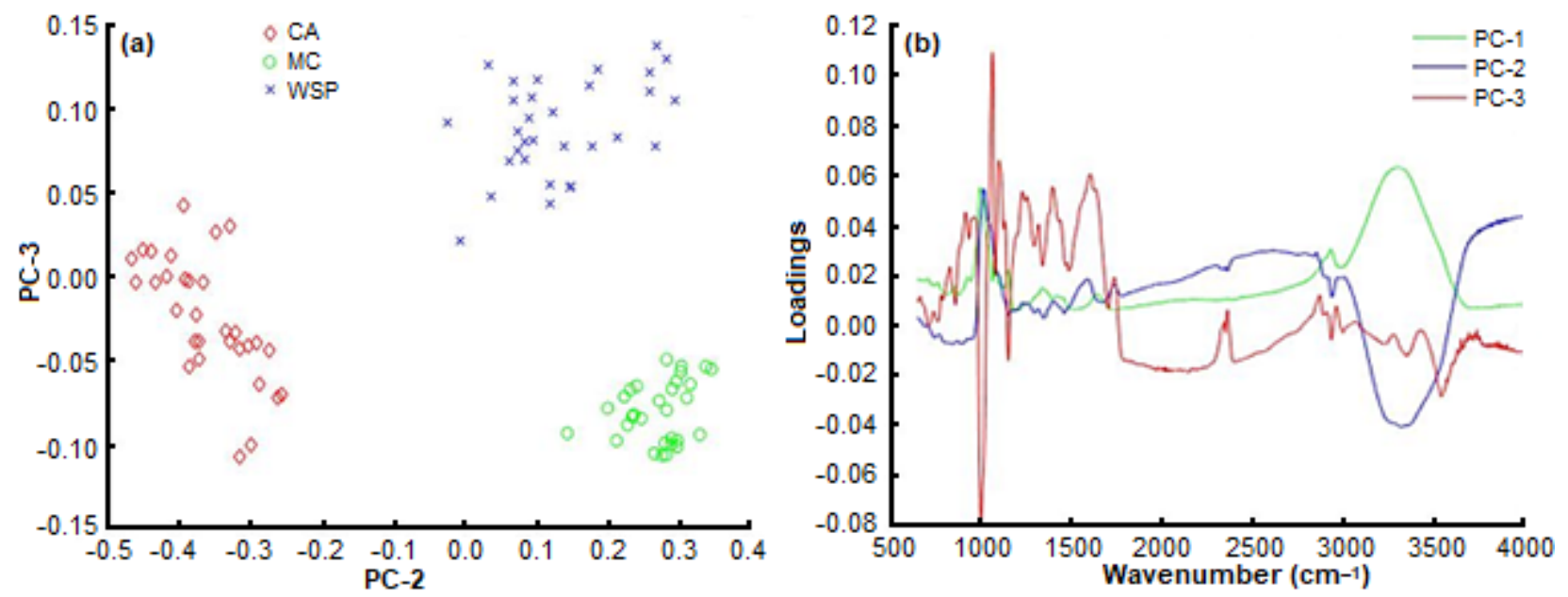

Fig 3. PCA of FT-IR spectra of CA (Canna edulis), MC (modified cassava), and WSP (white sweet potato): (a) score plot and (b) loadings profile of PC-2 and PC-3 
loading plot (Fig. 2(b)) shows the highest value for PC-1 at $5208 \mathrm{~cm}^{-1}$ corresponding to $\mathrm{CO}$ stretch second overtone assigned to CONH structure and $6944 \mathrm{~cm}^{-1} \mathrm{CH}$ combination corresponding to $\mathrm{CH}_{2}$. On the other hand, PC-2 shows the peaks at $5208 \mathrm{~cm}^{-1}$ similar to that of PC1, at which $7184 \mathrm{~cm}^{-1}$ is $\mathrm{CH}$ combination of $\mathrm{CH}_{2}$ [22].

Fig. 3(b) shows a clear separation of three types of flour indicated by PC-2 and PC-3 for FT-IR data, which account for $13 \%$ of the total variability, but in total, the first three principal components account for $99.7 \%$ the total variability. The loadings of PC-2 (Fig. 3(b)) showed a negative influence of the bands at $3304 \mathrm{~cm}^{-1}$ associated with $\mathrm{NH}$ of amines and $\mathrm{OH}$ of alcohols, phenols, carboxylic acids, and water, as well as at $1018 \mathrm{~cm}^{-1}$ associated to $\mathrm{CO}$ of alcohols. The PC-3 shows several peaks on the fingerprint region, which shows several absorption peaks between $1000-1300 \mathrm{~cm}^{-1}$, which are assigned to $\mathrm{CO}$ stretch of esters, ethers, alcohols, and carboxylic acids, as well as at $1600 \mathrm{~cm}^{-1}$ assigned to CC of the aromatic bond.

\section{Partial Least Square Discriminant Analysis Model}

A PLS-DA model is used to distinguish three types of tuber flour that have a similar color. The PLS-DA models are developed using the preprocessed spectra of samples from FT-NIR and FT-IR spectroscopy. The PLSDA calibration model classified Canna edulis, modified cassava, and white sweet potato flour with $100 \%$ accuracy for calibration and predictive model for both FT-NIR and
FT-IR spectroscopy (data not shown), by using original spectra and applying all preprocessed methods, from normalization to derivatives. The high classification accuracy demonstrates that tuber flours having a similar color can be correctly identified by FT-NIR and FT-IR instruments.

The highest coefficient determination and standard error of prediction (SEP) of prediction, which are $\mathrm{R}^{2}$ of 0.99 and 0.012 , are obtained by applying the Savitzky-Golay $1^{\text {st }}$ derivative preprocessed for FT-NIR spectra. For FT-IR, the coefficient determination and SEP of prediction, which are $\mathrm{R}^{2}$ of 0.99 and 0.042 , are obtained by also applying the Savitzky-Golay $1^{\text {st }}$ derivative preprocessed. The $1^{\text {st }}$ derivative provided the best PLS model since it removed the baseline offset and provided lesser peaks compared to other derivatives but was able to be distinctly observed. The PLS-DA results are listed in Table 1 for the FT-NIR and FT-IR spectral data.

Fig. 4 shows the beta coefficient of the PLS-DA model resulted from the Savitzky-Golay $1^{\text {st }}$ derivative of FT-NIR and FT-IR spectra, which give the best calibration and prediction model for discriminating Canna edulis, modified cassava, and sweet potato flour. Several distinct peaks can be observed on FT-NIR spectra (Fig. 4(a)) at $4048 \mathrm{~cm}^{-1}$ symmetrical $\mathrm{CNC}$ stretch first overtone relating to protein, $4404 \mathrm{~cm}^{-1} \mathrm{OH}$ stretch or $\mathrm{CO}$ stretch combination relating to cellulose, $5172 \mathrm{~cm}^{-1} \mathrm{OH}$ stretch or $\mathrm{HOH}$ deformation combination relating to starch [22],
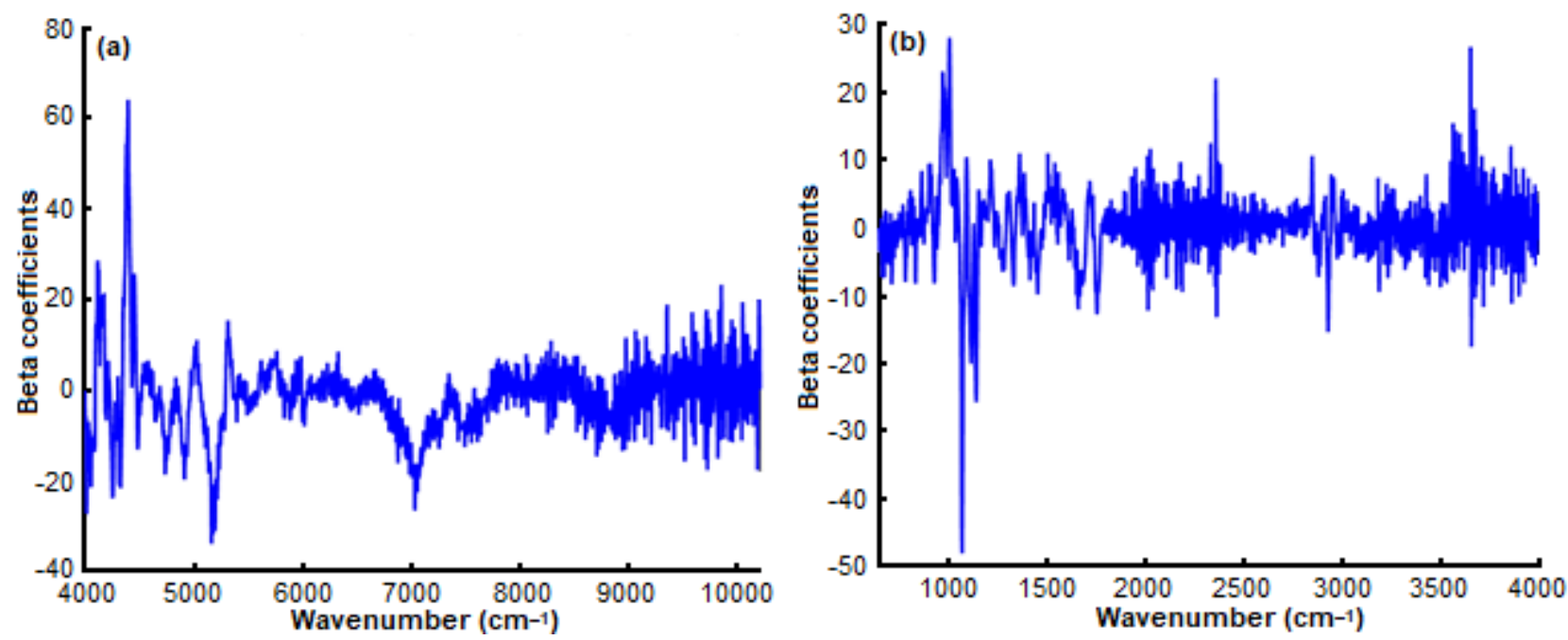

Fig 4. Beta coefficients of Savitzky-Golay $1^{\text {st }}$ derivative of (a) FT-NIR spectra and (b) FT-IR spectra 
Table 1. Comparison of original PLS-DA model versus VIP model with various preprocessing methods on FT-NIR and FT-IR spectra

\begin{tabular}{|c|c|c|c|c|c|c|c|c|c|c|c|}
\hline \multirow{3}{*}{ Spectroscopy } & \multirow{3}{*}{ Preprocessing } & \multicolumn{5}{|c|}{ PLS-DA } & \multicolumn{4}{|c|}{ PLS-DA VIP } & \multirow{3}{*}{ LV } \\
\hline & & \multicolumn{2}{|c|}{ Calibration } & \multicolumn{2}{|c|}{ Prediction } & \multirow{2}{*}{ LV } & \multicolumn{2}{|c|}{ Calibration } & \multicolumn{2}{|c|}{ Prediction } & \\
\hline & & $\mathrm{R}^{2}$ & SEC & $\mathrm{R}^{2}$ & SEP & & $\mathrm{R}^{2}$ & SEC & $\mathrm{R}^{2}$ & SEP & \\
\hline \multirow[t]{9}{*}{ FT-NIR } & Mean norm & 0.994 & 0.062 & 0.994 & 0.062 & 5 & 0.994 & 0.066 & 0.988 & 0.091 & 4 \\
\hline & Max norm & 0.994 & 0.065 & 0.994 & 0.065 & 5 & 0.992 & 0.072 & 0.987 & 0.096 & 4 \\
\hline & Range norm & 0.995 & 0.060 & 0.995 & 0.060 & 5 & 0.996 & 0.054 & 0.991 & 0.080 & 6 \\
\hline & MSC & 0.998 & 0.040 & 0.998 & 0.040 & 7 & 0.997 & 0.045 & 0.995 & 0.060 & 6 \\
\hline & SNV & 0.997 & 0.043 & 0.997 & 0.043 & 7 & 0.996 & 0.051 & 0.995 & 0.060 & 6 \\
\hline & SG1 & 0.999 & 0.012 & 0.999 & 0.012 & 5 & 0.998 & 0.034 & 0.996 & 0.050 & 5 \\
\hline & SG2 & 0.999 & 0.0121 & 0.999 & 0.0121 & 5 & 0.998 & 0.033 & 0.995 & 0.057 & 5 \\
\hline & Original & 0.998 & 0.0409 & 0.998 & 0.0409 & 6 & 0.998 & 0.039 & 0.995 & 0.060 & 5 \\
\hline & Variables & & \multicolumn{3}{|c|}{1557} & \multicolumn{6}{|c|}{385} \\
\hline \multirow[t]{9}{*}{ FT-IR } & Mean norm & 0.995 & 0.059 & 0.995 & 0.059 & 5 & 0.99 & 0.08 & 0.98 & 0.11 & 5 \\
\hline & Max norm & 0.994 & 0.066 & 0.994 & 0.066 & 5 & 0.99 & 0.07 & 0.99 & 0.09 & 6 \\
\hline & Range norm & 0.987 & 0.097 & 0.987 & 0.097 & 3 & 0.97 & 0.14 & 0.96 & 0.18 & 3 \\
\hline & MSC & 0.995 & 0.061 & 0.995 & 0.061 & 5 & 0.99 & 0.08 & 0.99 & 0.10 & 5 \\
\hline & SNV & 0.995 & 0.059 & 0.995 & 0.059 & 5 & 0.99 & 0.07 & 0.99 & 0.09 & 5 \\
\hline & SG1 & 0.998 & 0.042 & 0.998 & 0.042 & 5 & 0.99 & 0.06 & 0.96 & 0.17 & 6 \\
\hline & SG2 & 0.995 & 0.057 & 0.995 & 0.057 & 5 & 0.99 & 0.06 & 0.95 & 0.19 & 6 \\
\hline & Original & 0.986 & 0.100 & 0.986 & 0.100 & 5 & 0.98 & 0.11 & 0.95 & 0.19 & 5 \\
\hline & Variables & \multicolumn{4}{|c|}{1738} & \multicolumn{6}{|c|}{608} \\
\hline
\end{tabular}

Note: $\mathrm{R}^{2}$ = coefficient of determination; SEC = standard error of calibration' SEP = standard error of prediction; LV = latent variable; Mean norm = mean normalization; Max norm = maximum normalization; Range norm = range normalization; MSC = multiplicative scatter correction; SNV = standard normal variate, SG1 = Savitzky-Golay $1^{\text {st }}$ derivative; SG2 = Savitzky-Golay $2^{\text {rd }}$ derivative

and $7040 \mathrm{~cm}^{-1}$ relating to water [23]. Results indicate that starch, protein, cellulose, and water may contribute to flour discrimination based on FT-NIR spectra.

Clear peaks in the FT-IR region (Fig. 4(b)) can be observed at 3330, 2929, and $1641 \mathrm{~cm}^{-1}$, which are related to water and protein absorption bands [24]. Other bands below $1500 \mathrm{~cm}^{-1}$ contain complex spectra, but it can be observed that at $1078 \mathrm{~cm}^{-1}$ are due to $\mathrm{CC}$ bond vibrations as well as 1011 and $1144 \mathrm{~cm}^{-1}$ are due to COC stretching bonds relate to the carbohydrate [25]. The distinct peak at $3649 \mathrm{~cm}^{-1}$ indicates the $\mathrm{OH}$ stretching bond relates to water. Those imply that carbohydrate and water contents among samples contribute to the discrimination of samples based on FT-IR spectra.

FT-NIR and FT-IR spectra contain lots of variables (in this study, there are 1557 and 1738 variables), although only parts of the variables are related to the property of interests. Therefore, the variable selection method is used to select the number of variables that are able to improve the performance of the model and to ease the interpretation [26]. The variable selection used in this study is the variable importance on projection (VIP), which considers all variables for initialization and then filters the unimportant variables based on some criterions [26]. VIP cut off used is 1.0. The new PLS-DA model developed using VIP selection is compared with the original PLS-DA model, as shown in Table 1. VIP selection is able to reduce variables from 1557 to 385 for FT-NIR and 1738 to 608 for FT-IR. The VIP-PLS-DA model achieves $\mathrm{R}^{2}$ of 0.99 and SEP of $0.05 \%$ for FT NIR with the Savitzky-Golay $1^{\text {st }}$ derivative preprocessed spectra, as well as $\mathrm{R}^{2}$ of 0.99 and SEP of $0.09 \%$ for FT IR with the maximum normalization data preprocessing methods. The VIP selection will provide a faster process and lesser computer memory required for computation. 


\section{- CONCLUSION}

This study aimed to differentiate three types of tuber flours made of Canna edulis, modified cassava, and white sweet potato. The PLS-DA model achieved $100 \%$ accuracy in predicting all types of flours, both with a coefficient of determination $\left(\mathrm{R}^{2}\right)$ of 0.99 and a standard error of prediction (SEP) of $0.03 \%$ for FT-NIR and $\mathrm{R}^{2}$ of 0.99 and SEP of $0.08 \%$ for FT-IR. The VIP method is used to develop PLS-DA models, which result in reducing variables from 1738 to 608 variables for FT-IR and from 1557 to 385 variables for FT-NIR. The VIP-PLS-DA also achieves high accuracy models with $\mathrm{R}^{2}$ of 0.99 and SEP of $0.09 \%$ as well as $\mathrm{R}^{2}$ of 0.99 and SEP of $0.05 \%$ for FT-IR and FT-NIR, respectively. Both FT-NIR and FT-IR provide high accuracy and fast methods in the discriminating types of tuber flours that have similar color, which will be beneficial for large samples.

\section{- ACKNOWLEDGMENTS}

This work is done at and supported by the Department of Biosystems Machinery Engineering, College of Agricultural and Life Science, Chungnam National University, Republic of Korea. Great appreciation is delivered to all members of the Nondestructive Bio-Sensing Laboratory for their support and assistance in finishing this work.

\section{- REFERENCES}

[1] Chandrasekara, A., and Kumar, T.J., 2016, Roots and tuber crops as functional foods: A review on phytochemical constituents and their potential health benefits, Int. J. Food Sci., 2016, 3631647.

[2] More, S.J., Ravi, V., and Raju, S., 2019, "Tropical tuber crops" in Postharvest Physiological Disorders in Fruits and Vegetables, $1^{\text {st }}$ Ed., Eds. de Freitas, S.T., and Pareek, S., CRC Press., 719-758.

[3] Manning, L., 2016, Food fraud: Policy and food chain, Curr. Opin. Food Sci., 10, 16-21.

[4] Starr, G., Bredie, W.L.P., and Hansen, Å.S., 2013, Sensory profiles of cooked grains from wheat species and varieties, J. Cereal Sci., 57 (3), 295-303.

[5] Pico, J., Tapia, J., Bernal, J., and Gómez, M., 2018, Comparison of different extraction methodologies for the analysis of volatile compounds in gluten-free flours and corn starch by GC/QTOF, Food Chem., 267, 303-312.

[6] Hidalgo, A., Fongaro, L., and Brandolini, A., 2017, Colour screening of whole meal flours and discrimination of seven Triticum subspecies, $J$. Cereal Sci., 77, 9-16.

[7] Aprianita, A., Vasiljevic, T., Bannikova, A., and Kasapis, S., 2014, Physicochemical properties of flours and starches derived from traditional Indonesian tubers and roots, J. Food Sci. Technol., 51 (12), 3669-3679.

[8] Esteki, M., Simal-Gandara, J., Shahsavari, Z., Zandbaaf, S., Dashtaki, E., and Heyden, Y.V., 2018, A review on the application of chromatographic methods, coupled to chemometrics, for food authentication, Food Control, 93, 165-182.

[9] Manley, M., 2014, Near-infrared spectroscopy and hyperspectral imaging: Non-destructive analysis of biological materials, Chem. Soc. Rev., 43 (24), 82008214.

[10] Shi, H., Lei, Y., Prates, L.L., and Yu, P., 2019, Evaluation of near-infrared (NIR) and Fourier transform mid-infrared (ATR-FT/MIR) spectroscopy techniques combined with chemometrics for the determination of crude protein and intestinal protein digestibility of wheat, Food Chem., 272, 507-513.

[11] Chen, J., Zhu, S., and Zhao, G., 2017, Rapid determination of total protein and wet gluten in commercial wheat flour using siSVR-NIR, Food Chem., 221, 1939-1946.

[12] Sampaio, P.S., Soares, A., Castanho, A., Almeida, A.S., Oliveira, J., and Brites, C., 2018, Optimization of rice amylose determination by NIR-spectroscopy using PLS chemometrics algorithms, Food Chem., 242, 196-204.

[13] Lebot, V., Champagne, A., Malapa, R., and Shiley, D., 2009, NIR determination of major constituents in tropical root and tuber crop flours, J. Agric. Food Chem., 57 (22), 10539-10547.

[14] Ding, X., Ni, Y., and Kokot, S., 2015, NIR spectroscopy and chemometrics for the 
discrimination of pure, powdered, purple sweet potatoes and their samples adulterated with the white sweet potato flour, Chemom. Intell. Lab. Syst., 144, $17-23$.

[15] Li, X., Lu, R., Wang, Z., Wang, P., Zhang, L., and Jia, P., 2018, Detection of corn and whole wheat adulteration in white pepper powder by near infrared spectroscopy, Am. J. Food Sci. Technol., 6 (3), 114-117.

[16] Chen, H., Tan, C., Lin, Z., and Li, H., 2019, Quantifying several adulterants of notoginseng powder by near-infrared spectroscopy and multivariate calibration, Spectrochim. Acta, Part A, 211, 280-286.

[17] Ding, X., Ni, Y., and Kokot, S., 2015, NIR spectroscopy and chemometrics for the discrimination of pure, powdered, purple sweet potatoes and their samples adulterated with the white sweet potato flour, Chemom. Intell. Lab. Syst., 144, 17-23.

[18] Giraudo, A., Grassi, S., Savorani, F., Gavoci, G., Casiraghi, E., and Geobaldo, F., 2019, Determination of the geographical origin of green coffee beans using NIR spectroscopy and multivariate data analysis, Food Control, 99, 137-145.

[19] Agelet, L.E., and Hurburgh, C.R., 2010, A tutorial on near infrared spectroscopy and its calibration, Crit. Rev. Anal. Chem., 40 (4), 246-260.

[20] Jawaid, S., Talpur, F.N., Sherazi, S.T.H., Nizamani, S.M., and Khaskheli, A.A., 2013, Rapid detection of melamine adulteration in dairy milk by SB-ATRFourier transform infrared spectroscopy, Food
Chem., 141 (3), 3066-3071.

[21] Hell, J., Prückler, M., Danner, L., Henniges, U., Apprich, S., Rosenau, T., Kneifel, W., and Böhmdorfer, S., 2016, A comparison between nearinfrared (NIR) and mid-infrared (ATR-FTIR) spectroscopy for the multivariate determination of compositional properties in wheat bran samples, Food Control, 60, 365-369.

[22] Aenugu, H.P.R., Kumar, D.S., Srisudharson, Parthiban, N., Ghosh, S.S., and Banji, D., 2011, Near infra red spectroscopy- An overview, Int. J. ChemTech Res., 3 (2), 825-836.

[23] Shi, H., and Yu, P., 2017, Comparison of gratingbased near-infrared (NIR) and Fourier transform mid-infrared (ATR-FT/MIR) spectroscopy based on spectral preprocessing and wavelength selection for the determination of crude protein and moisture content in wheat, Food Control, 82, 57-65.

[24] Kong, J., and Yu, S., 2007, Fourier transform infrared spectroscopic analysis of protein secondary structures, Acta Biochim. Biophys. Sin., 39 (8), 549559.

[25] Lohumi, S., Lee, S., Lee, W.H., Kim, M.S., Mo, C., Bae, H., and Cho, B.K., 2014, Detection of starch adulteration in onion powder by FT-NIR and FTIR spectroscopy, J. Agric. Food Chem., 62 (38), 9246-9251.

[26] Yun, Y.H., Li, H.D., Deng, B.C., and Cao, D.S., 2019, An overview of variable selection methods in multivariate analysis of near-infrared spectra, TrAC, Trends Anal. Chem., 113, 102-115. 